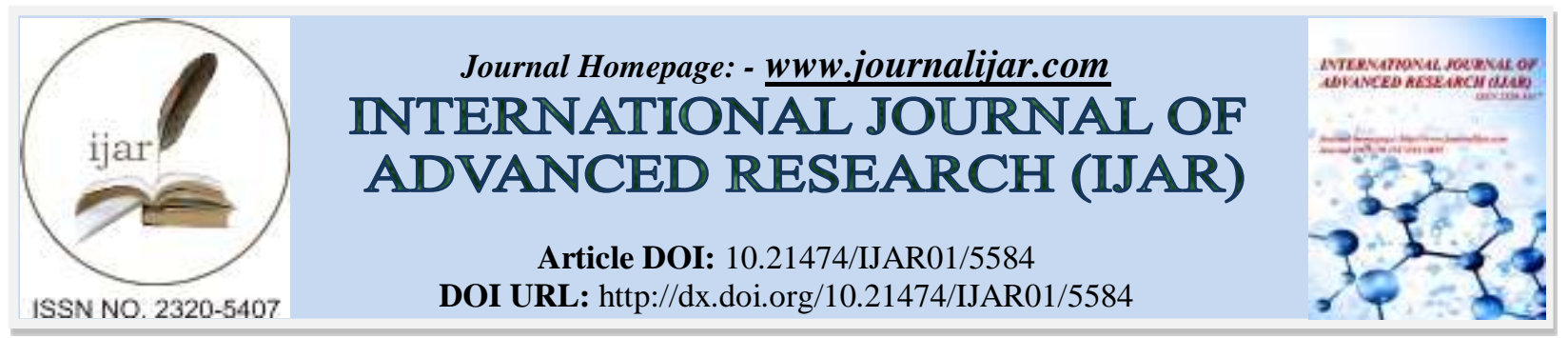

RESEARCH ARTICLE

\title{
COMPARATIVE STUDY OF DDEs AND ODEs.
}

Subhransu Sundar Das ${ }^{1}$, Dhirendra Kumar Dalai ${ }^{2}$ and Purna Chandra Nayak ${ }^{3}$.

1. Department of Mathematics, Semiliguda College, Semiliguda, Koraput-764036, Odisha, India.

2. Department of Mathematics, Govt. (Jr) Science College, Ayeba, Kendrapara, Odisha, India.

3. Department of Mathematics, Bhadrak (Auto) College, Bhadrak, Odisha, India.

\section{Manuscript Info}

Manuscript History

Received: 10 August 2017

Final Accepted: 12 September 2017

Published: October 2017

Key words:-

Characteristics Equation, Collocation

Method, DDEs, ODEs, Order failure.

\section{Abstract}

A comparative study of Delay Differential Equations (DDEs) and Ordinary Differential Equations (ODEs) have been reflected in our present paper. In this paper we explain :

(i) How Delay Differential Equation (DDE) differ from Ordinary Differential Equation (ODE) on the basis of time.

(ii) The solution of DDEs using method of steps (MOS) and how the DDEs are solved by converting it to ODEs.

(iii) The equivalency of some DDEs to ODEs and how to find characteristics equation of Delay Differential Equations (DDEs)

(iv) The order failure of DDEs using Gaussian collocation method for $\mathrm{v}$ $=1$ and complicacy arises when $\mathrm{v}>1$.

Copy Right, IJAR, 2017,. All rights reserved.

\section{Introduction:-}

All processes take time to complete, while physical process i.e, acceleration and deceleration take little time compared to the times needed to travel most distances, the times involved in biological process such as gestation and maturation can be considerable when compared to the data collection times in most population studies. So, it is essential to includes these process of times into mathematical models in population dynamics. The process of times are called delay times and the model that include such delay times is Delay Differential Equation (DDE) models.

Delay Differential Equations (DDEs) are a type of D.E. in which the derivative of the unknown function at a certain time is given in terms of the values of the function at previous times, but in Ordinary Differential Equations (ODEs), the derivative of the unknown function depends upon a particular time.

DDEs are also called time-delay systems, systems with aftereffect or dead time, hereditary systems, equations with deviating argument, or differential-difference equations.

A general form of the time-delay differential equation for the unknown function $x(t) \in R^{n}$ is $\frac{d}{d t} x(t)=f\left(t, x(t), x_{t}\right)$

Where $\mathrm{X}_{\mathrm{t}}=\{\mathrm{X}(\tau): \tau \leq \mathrm{t}\}$ represents the trajectory of the solution in the past.

In this equation, $f$ is a functional operator from $\operatorname{RXR}^{n} X C^{1}\left(R, R^{n}\right)$ to $R^{n}$. 


\section{Examples of Delay Differential Equations:-}

1. Continuous DDE is of the form

$$
\frac{\mathrm{d}}{\mathrm{dt}} \mathrm{x}(\mathrm{t})=\mathrm{f}\left(\mathrm{t}, \mathrm{x}(\mathrm{t}), \int_{-\infty}^{0} \mathrm{x}(\mathrm{t}+\tau) \mathrm{d} \mu(\tau)\right)
$$

2. Discrete DDE is of the form

$$
\frac{\mathrm{d}}{\mathrm{dt}} \mathrm{x}(\mathrm{t})=\mathrm{f}\left(\mathrm{t}, \mathrm{x}(\mathrm{t}), \mathrm{x}\left(\mathrm{t}-\tau_{1}\right), \ldots ., \mathrm{x}\left(\mathrm{t}-\tau_{\mathrm{m}}\right)\right), \text { where } \tau_{1}>\tau_{2}>\ldots>\tau_{\mathrm{m}} \geq 0
$$

3. Linear with discrete delays is of form

$$
\frac{d}{d t} x(t)=a_{0} x(t)+a_{1} x\left(t-\tau_{1}\right)+\ldots .+a_{m} x\left(t-\tau_{m}\right), \text { where } a_{0}, a_{1}, \ldots, a_{m} \in R^{n \times n}
$$

4. DDE of the form

$$
\frac{d}{d t} x(t)=a x(t)+b x(\lambda t) \text { where } a, b \text { and } \lambda \text { are constants and } 0<\lambda<1 \text { is called pantograph equation. }
$$

\section{Solution of Delay Differential Equations (DDEs):-}

From an ODEs, a unique solution is determined by an initial point at an initial time $\mathrm{t}_{0}$, but for a DDE, one requires information on the entire interval $\left[\mathrm{t}_{0}-\tau, \mathrm{t}_{0}\right], \mathrm{t}_{0}-\tau$ is the previous time i.e., to know rate of change at $\mathrm{t}_{0}$, one needs $\mathrm{x}\left(\mathrm{t}_{0}\right)$ and $\mathrm{x}\left(\mathrm{t}_{0}-\tau\right)$.

DDEs are mostly solved stepwise fashion with a principle called the Method Of Steps (MOS).

\section{For example:-}

Consider the DDE with a single delay

$$
\frac{\mathrm{d}}{\mathrm{dt}} \mathrm{x}(\mathrm{t})=\mathrm{f}(\mathrm{x}(\mathrm{t}), \mathrm{x}(\mathrm{t}-\tau)), \mathrm{t} \in[0, \tau] \text {, with given initial condition } \phi:[-\tau, 0] \rightarrow \mathrm{R}^{\mathrm{n}}
$$

Then the solution on the interval $[0, \tau]$ is given by

$\psi(\mathrm{t})$ which is the solution to the non-homogeneous initial value problem

$\frac{\mathrm{d}}{\mathrm{dt}} \psi(\mathrm{t})=\mathrm{f}(\psi(\mathrm{t}), \phi(\mathrm{t}-\tau))$ with

initial condition

$\psi(0)=\phi(0)$

This can be continued for the successive intervals by using the solution to the previous interval as non-homogeneous term. In general, the initial value problem is often solved numerically.

\section{Examples:-}

1. Let

$$
\begin{aligned}
& f(x(t), x(t-\tau))=a x(t-\tau), \phi(t)=1, t \in[-\tau, 0] \\
& \text { i.e, } \frac{d}{d t} x(t)=a x(t-\tau), t \in[0, \tau], x(t)=\phi(t), t \in[-\tau, 0]
\end{aligned}
$$

Solution in the interval $[0, \tau]$ i.e, for $t \in[0, \tau]$ is given by

$$
\begin{array}{r}
x(t)=x(0)+\int_{s=0}^{t} \frac{d}{d t} x(s) d s \\
=1+\int_{s=0}^{t} a x(s-\tau) d s
\end{array}
$$




$$
\begin{aligned}
& =1+\int_{\mathrm{s}=0}^{\mathrm{t}} \mathrm{a} \phi(\mathrm{s}-\tau) \mathrm{ds} \\
& =1+\mathrm{a} \int_{\mathrm{s}=0}^{\mathrm{t}} 1 \mathrm{ds} \\
& =1+\mathrm{a}[\mathrm{s}]_{0}^{\mathrm{t}}=1+\mathrm{at}
\end{aligned}
$$

i.e, $x(t)=1+$ at, with the initial condition

$\mathrm{x}(0)=\phi(0)=1$

Again, for interval $\mathrm{t} \in[\tau, 2 \tau]$, we integrate and fit the initial condition,

$x(t)=x(\tau)+\int_{s=\tau}^{t} \frac{d}{d t} x(s) d s$

$=(1+a \tau)+\int_{s=\tau}^{t} \mathrm{ax}(\mathrm{s}) \mathrm{ds}$

$=(1+a \tau)+a \int_{s=\tau}^{t} x(s-\tau) d s$

$=(1+\mathrm{a} \tau)+\mathrm{a} \int_{\mathrm{s}=\tau}^{\mathrm{t}}\{1+\mathrm{a}(\mathrm{s}-\tau)\} \mathrm{ds}$, Put $\mathrm{s}-\tau=\mathrm{z}, \mathrm{ds}=\mathrm{dz}$; when $\mathrm{s}=\tau, \mathrm{z}=0 ; \mathrm{s}=\mathrm{t}, \mathrm{z}=\mathrm{t}-\tau$

$=(1+a \tau)+a \int_{0}^{t-\tau}(1+a z) d z$

$=(1+a \tau)+a\left[z+a \frac{z^{2}}{2}\right]_{0}^{t-\tau}$

$=(1+a \tau)+a\left[(t-\tau)+a \frac{(t-\tau)^{2}}{2}\right]$

i.e., $x(t)=(1+a \tau)+a(t-\tau)\left[\frac{a}{2}(t-\tau)+1\right]$

with the initial condition $\mathrm{x}(\tau)=\phi(\tau)=1+\mathrm{a} \tau$,

and this can be continued for the successive intervals by using the solution to the previous interval.

\section{Reduction of DDE to ODE:-}

In some cases, delay differential equations are equivalent to a system of ODEs.

2. The DDE

$$
\begin{aligned}
& \frac{d}{d t} x(t)=f\left(t, x(t), \int_{-\infty}^{0} x(t+\tau) e^{\lambda \tau} d \tau\right), \text { can be converted to ODE by choosing, } \\
& y(t)=\int_{-\infty}^{0} x(t+\tau) e^{\lambda \tau} d \tau \\
& \frac{d y}{d t}=\int_{-\infty}^{0} x^{\prime}(t+\tau) e^{\lambda \tau} d \tau
\end{aligned}
$$




$$
\begin{aligned}
& \left.=\mathrm{e}^{\lambda \tau} \mathrm{x}(\mathrm{t}+\tau)\right]_{-\infty}^{0}-\int_{-\infty}^{0} \lambda \mathrm{e}^{\lambda \tau} \mathrm{x}(\mathrm{t}+\tau) \mathrm{d} \tau \\
& =(\mathrm{x}(\mathrm{t})-0))-\lambda \int_{-\infty}^{0} \mathrm{e}^{\lambda \tau} \mathrm{x}(\mathrm{t}+\tau) \mathrm{d} \tau \\
& =\mathrm{x}(\mathrm{t})-\lambda \mathrm{y} \\
& \therefore \frac{\mathrm{dy}}{\mathrm{dt}}=\mathrm{x}-\lambda \mathrm{y}
\end{aligned}
$$

So we get a system of ODEs

$$
\frac{d}{d t} x(t)=f(t, x, y), \frac{d}{d t} y(t)=x-\lambda y
$$

3. The DDE

$$
\frac{d}{d t} x(t)=f\left(t, x(t), \int_{-\infty}^{0} x(t+\tau) \cos (\alpha \tau+\beta) d \tau\right)
$$

is converted to ODE by,

Letting $y(t)=\int_{-\infty}^{0} x(t+\tau) \cos (\alpha \tau+\beta) d \tau$

$\frac{\mathrm{dy}}{\mathrm{dt}}=\int_{-\infty}^{0} \mathrm{x}^{\prime}(\mathrm{t}+\tau) \cos (\alpha \tau+\beta) \mathrm{d} \tau$

$\left.\Rightarrow \frac{\mathrm{dy}}{\mathrm{dt}}=\cos (\alpha \tau+\beta) \mathrm{x}(\mathrm{t}+\tau)\right]_{-\infty}^{0}+\int_{-\infty}^{0} \alpha \sin (\alpha \tau+\beta) \mathrm{x}(\mathrm{t}+\tau) \mathrm{d} \tau$

$=x(t) \cos \beta+\alpha \int_{-\infty}^{0} \sin (\alpha \tau+\beta) x(t+\tau) d \tau$

$\Rightarrow \frac{\mathrm{d}}{\mathrm{dt}} \mathrm{y}(\mathrm{t})=\mathrm{x}(\mathrm{t}) \cos \beta+\alpha \mathrm{z}$

Where $z=\int_{-\infty}^{0} \sin (\alpha \tau+\beta) x(t+\tau) d \tau$, i.e, $z=\int_{-\infty}^{0} x(t+\tau) \sin (\alpha \tau+\beta) d \tau$

Also $z=\int_{-\infty}^{0} x(t+\tau) \sin (\alpha \tau+\beta) d \tau$

$\frac{\mathrm{dz}}{\mathrm{dt}}=\int_{-\infty}^{0} \mathrm{x}^{\prime}(\mathrm{t}+\tau) \sin (\alpha \tau+\beta) \mathrm{dt}$

$\left.\Rightarrow \frac{\mathrm{dz}}{\mathrm{dt}}=\sin (\alpha \tau+\beta) \mathrm{x}(\mathrm{t}+\tau)\right]_{-\infty}^{0}-\int_{-\infty}^{0} \alpha \cos (\alpha \tau+\beta) \mathrm{x}(\mathrm{t}+\tau) \mathrm{d} \tau$

$=x(t) \sin \beta-\alpha \int_{-\infty}^{0} \cos (\alpha \tau+\beta) x(t+\tau) d \tau$

$=x(t) \sin \beta-\alpha y$

So the given DDE is equivalent to

$$
\frac{d}{d t} x(t)=f(t, x, y), \frac{d}{d t} y(t)=\cos (\beta) x+\alpha z,
$$




$$
\begin{aligned}
& \frac{d}{d t} z(t)=\sin (\beta) x-\alpha y \\
& \text { Where } \\
& y=\int_{-\infty}^{0} x(t+\tau) \cos (\alpha \tau+\beta) d \tau, \quad z=\int_{-\infty}^{0} x(t+\tau) \sin (\alpha \tau+\beta) d \tau
\end{aligned}
$$

The Characteristic Equation:-

As in case of ODEs, many properties of linear DDEs can be characterized and analyzed using the characteristic equation.

To find the characteristic equation associated with the linear DDE with discrete delays

$$
\begin{aligned}
& \frac{\mathrm{d}}{\mathrm{dt}} \mathrm{x}(\mathrm{t})=\mathrm{a}_{0} \mathrm{x}(\mathrm{t})+\mathrm{a}_{1} \mathrm{x}\left(\mathrm{t}-\tau_{1}\right)+\ldots .+\mathrm{a}_{\mathrm{m}} \mathrm{x}\left(\mathrm{t}-\tau_{\mathrm{m}}\right) \\
& \text { Let } \mathrm{x}(\mathrm{t})=\mathrm{e}^{\lambda \mathrm{t}} \\
& \Rightarrow x\left(t-\tau_{i}\right)=e^{\lambda\left(t-\tau_{i}\right)}, i=1,2, \ldots, m
\end{aligned}
$$

then the above DDE reduces to

$$
\begin{aligned}
& \frac{d}{d t} e^{\lambda t}=a_{0} e^{\lambda t}+a_{1} e^{\lambda\left(t-\tau_{1}\right)}+\ldots+a_{m} e^{\lambda\left(t-\tau_{m}\right)} \\
& \Rightarrow \lambda \mathrm{e}^{\lambda t}=\mathrm{e}^{\lambda t}\left(\mathrm{a}_{0}+\mathrm{a}_{1} \mathrm{e}^{-\lambda \tau_{1}}+\ldots .+\mathrm{a}_{\mathrm{m}} \mathrm{e}^{-\lambda \tau_{\mathrm{m}}}\right) \\
& \Rightarrow \lambda=\left(\mathrm{a}_{0}+\mathrm{a}_{1} \mathrm{e}^{-\lambda \tau_{1}}+\ldots .+\mathrm{a}_{\mathrm{m}} \mathrm{e}^{-\lambda \tau_{\mathrm{m}}}\right), \mathrm{e}^{\lambda \mathrm{t}} \neq 0 \\
& \Rightarrow \lambda \mathrm{I}=\left(\mathrm{a}_{0}+\mathrm{a}_{1} \mathrm{e}^{-\lambda \tau_{1}}+\ldots .+\mathrm{a}_{\mathrm{m}} \mathrm{e}^{-\lambda \tau_{\mathrm{m}}}\right) \\
& \Rightarrow\left[-\lambda \mathrm{I}+\mathrm{a}_{0}+\mathrm{a}_{1} \mathrm{e}^{-\lambda \tau_{1}}+\ldots .+\mathrm{a}_{\mathrm{m}} \mathrm{e}^{-\lambda \tau_{\mathrm{m}}}\right]=0 \\
& \Rightarrow \operatorname{det}\left(-\lambda \mathrm{I}+\mathrm{a}_{0}+\mathrm{a}_{1} \mathrm{e}^{-\lambda \tau_{1}}+\ldots .+\mathrm{a}_{\mathrm{m}} \mathrm{e}^{-\lambda \tau_{\mathrm{m}}}\right)=0,
\end{aligned}
$$

Which is the characteristic equation associated with the above linear DDE with discrete delays.

The roots $\lambda$ of the characteristic equation are called characteristic roots or eigen values and the solution set is referred to as the spectrum. This characteristic equation is a non-linear eigen problem and there are many methods to compute numerically.

4. Finding the characteristic equation for DDE

$$
\frac{\mathrm{d}}{\mathrm{dt}} \mathrm{x}(\mathrm{t})=-\mathrm{x}(\mathrm{t}-1)
$$

The characteristic equation for the DDE :

let, $x(t)=e^{\lambda t}$

so $\frac{\mathrm{d}}{\mathrm{dt}} \mathrm{e}^{\lambda \mathrm{t}}=-\mathrm{e}^{\lambda(\mathrm{t}-1)}$

$\Rightarrow \lambda \mathrm{e}^{\lambda \mathrm{t}}=-\mathrm{e}^{\lambda \mathrm{t}} \mathrm{e}^{-\lambda}$

$\Rightarrow \lambda=-\mathrm{e}^{\lambda}$

$\Rightarrow-\lambda-\mathrm{e}^{-\lambda}=0$, which is the characteristics equation of the given DDE and there are infinite number of solutions to this equation for complex $\lambda$.

Also, $-\lambda-\mathrm{e}^{-\lambda}=0$

$\Rightarrow \lambda=-\mathrm{e}^{-\lambda}$

$\Rightarrow \lambda=(-1) \mathrm{e}^{-\lambda}$

$\Rightarrow \lambda=\mathrm{W}_{\mathrm{k}}(-1)$, where $\mathrm{W}_{\mathrm{k}}$ is the kth Branch of the Lambert $-\mathrm{W}$ Function. 


\section{Is ODE theory enough, for numerical solution of DDEs ?}

Consider the general constant delay differential equation

$$
\begin{cases}\mathrm{y}^{\prime}(\mathrm{t})=\mathrm{f}(\mathrm{t}, \mathrm{y}(\mathrm{t}), \mathrm{y}(\mathrm{t}-1)), & \mathrm{t} \geq 0 \\ \mathrm{y}(\mathrm{t})=\phi(\mathrm{t}), & \mathrm{t} \leq 0\end{cases}
$$

The most natural, but not unique approach solving (1) numerically is to assign integration steps less than or equal to delay $\tau=1$ and to integrate step by step the ODEs obtained from (1) by substituting the delayed term y(t-1) by a function $\eta(t-1)$, given according to the value of $t$, either by the initial function $\phi(t-1)$ or by continuous extension of the approximate solution previously computed by the method itself.

Continuing this process, at the $(\mathrm{n}+1)^{\mathrm{st}}$ step, the equation to be solved is

$\left\{\begin{array}{l}\mathrm{W}_{\mathrm{n}+1}^{\prime}(\mathrm{t})=\mathrm{f}\left(\mathrm{t}, \mathrm{W}_{\mathrm{n}+1}(\mathrm{t}), \mathrm{x}(\mathrm{t}-1)\right), \mathrm{t}_{\mathrm{n}} \leq \mathrm{t} \leq \mathrm{t}_{\mathrm{n}+1} \\ \mathrm{~W}_{\mathrm{n}+1}\left(\mathrm{t}_{\mathrm{n}}\right)=\mathrm{y}_{\mathrm{n}}\end{array}\right.$

Where $x(s)= \begin{cases}\phi(s) & \text { for } s \leq 0 \\ \eta(s) & \text { for } 0 \leq s \leq t_{n}\end{cases}$

The integration formula provides the value of $y_{n+1}$ and the approximate solution $\eta$ of (1) is then continued in $\left[\mathrm{t}_{\mathrm{n}}, \mathrm{t}_{\mathrm{n}+1}\right]$ in such a way that

$$
\eta\left(\mathrm{t}_{\mathrm{n}+1}\right)=\mathrm{y}_{\mathrm{n}+1}
$$

The numerical ODE method gives approximate values of the solution of nodal points only, where as the DDE methods will be based on continuous extensions of numerical ODE schemes. The presence of a delayed term can be drastically modify some boundedness or stability properties and in general, the dynamics of the simpler ODE models.

\section{Order Failure:-}

In order to illustrate the possible loss of accuracy, consider the class of constant coefficient linear equations.

$$
\begin{cases}y^{\prime}(t)=a y(t)-\frac{\pi}{2} e^{a} y(t-1), & t \geq 0 \\ y(t)=\phi(t)=e^{a t} \sin \left(\frac{\pi}{2} t\right), & t \leq 0\end{cases}
$$

whose solutions, $\mathrm{y}(\mathrm{t})=\mathrm{e}^{\mathrm{at}} \sin \left(\frac{\pi}{2} \mathrm{t}\right)$, are class of $\mathrm{c}^{\infty}$ in $[-1, \infty]$

To check, $y(t)=e^{a t} \sin \left(\frac{\pi}{2} t\right)$ is the solution,

$$
\begin{aligned}
\mathrm{y}(\mathrm{t}) & =\mathrm{e}^{\mathrm{at}} \sin \left(\frac{\pi}{2} \mathrm{t}\right) \\
y^{\prime}(t) & =a e^{a t} \sin \left(\frac{\pi}{2} t\right)-\frac{\pi}{2} e^{a} e^{a(t-1)} \sin \left(\frac{\pi}{2}(t-1)\right) \\
= & \mathrm{ae}^{\mathrm{at}} \sin \left(\frac{\pi}{2} \mathrm{t}\right)-\frac{\pi}{2} \mathrm{e}^{\mathrm{at}} \sin \left(\frac{\pi}{2} \mathrm{t}-\frac{\pi}{2}\right) \\
= & \mathrm{ae}^{\mathrm{at}} \sin \left(\frac{\pi}{2} \mathrm{t}\right)+\frac{\pi}{2} \mathrm{e}^{\mathrm{at}} \sin \left(\frac{\pi}{2}-\frac{\pi}{2} \mathrm{t}\right) \\
= & \mathrm{ae}^{\mathrm{at}} \sin \left(\frac{\pi}{2} \mathrm{t}\right)+\frac{\pi}{2} \mathrm{e}^{\mathrm{at}} \cos \left(\frac{\pi}{2} \mathrm{t}\right)
\end{aligned}
$$




$$
\begin{aligned}
& \frac{d y}{d t}=a e^{a t} \sin \left(\frac{\pi}{2} \mathrm{t}\right)+\frac{\pi}{2} \mathrm{e}^{\mathrm{at}} \cos \left(\frac{\pi}{2} \mathrm{t}\right) \\
& \Rightarrow \mathrm{y}=\mathrm{a} \int \mathrm{e}^{\mathrm{at}} \sin \left(\frac{\pi}{2} \mathrm{t}\right)+\frac{\pi}{2} \int \mathrm{e}^{\mathrm{at}}\left(\cos \frac{\pi}{2} \mathrm{t}\right) \mathrm{dt} \\
& \Rightarrow \mathrm{y}=\mathrm{a}\left[\frac{\mathrm{e}^{\mathrm{at}}}{\mathrm{a}^{2}+\frac{\pi^{4}}{4}}\left(\mathrm{a} \sin \frac{\pi}{2} \mathrm{t}-\frac{\pi}{2} \cos \frac{\pi}{2} \mathrm{t}\right)\right]+\frac{\pi}{2}\left[\frac{\mathrm{e}^{\mathrm{at}}}{\mathrm{a}^{2}+\frac{\pi^{2}}{4}}\left(\mathrm{a} \cos \frac{\pi}{2} \mathrm{t}+\frac{\pi}{2} \sin \frac{\pi}{2} \mathrm{t}\right)\right]
\end{aligned}
$$

Using $\int e^{a x} \cos b x d x=\frac{e^{a x}}{a^{2}+b^{2}}(a \cos b x-b \sin b x)$

$$
\int e^{a x} \sin b x d x=\frac{e^{a x}}{a^{2}+b^{2}}(a \sin b x-b \cos b x)
$$

$=\mathrm{a}\left[\frac{4 \mathrm{e}^{\mathrm{at}}}{4 \mathrm{a}^{2}+\pi^{2}}\left(\mathrm{a} \sin \frac{\pi}{2} \mathrm{t}-\frac{\pi}{2} \cos \frac{\pi}{2} \mathrm{t}\right)\right]+\frac{\pi}{2}\left[\frac{4 \mathrm{e}^{\mathrm{at}}}{4 \mathrm{a}^{2}+\pi^{2}}\left(\mathrm{a} \cos \frac{\pi}{2} \mathrm{t}+\frac{\pi}{2} \sin \frac{\pi}{2} \mathrm{t}\right)\right]$

$=\frac{4 \mathrm{e}^{\mathrm{at}}}{4 \mathrm{a}^{2}+\pi^{2}}\left[\left(\mathrm{a}^{2}+\frac{\pi^{2}}{4}\right) \sin \frac{\pi}{2} \mathrm{t}\right]$

$=\frac{4 \mathrm{e}^{\mathrm{at}}}{4 \mathrm{a}^{2}+\pi^{2}}\left[\left(\frac{4 \mathrm{a}^{2}+\pi^{2}}{4}\right) \sin \frac{\pi}{2} \mathrm{t}\right]$

$=\mathrm{e}^{\mathrm{at}} \sin \frac{\pi}{2} \mathrm{t}$, which satisfies

So, $y(t)=e^{a t} \sin \frac{\pi}{2} t$ is the solution.

According to (2), for $\mathrm{n}=0,1, \ldots$ we shall solve the ODE,

$\left\{\begin{array}{l}\mathrm{w}_{\mathrm{n}+1}^{\prime}(\mathrm{t})=\mathrm{aw}_{\mathrm{n}+1}(\mathrm{t})-\frac{\pi}{2} \mathrm{e}^{\mathrm{a}} \mathrm{x}(\mathrm{t}-1), \mathrm{t}_{\mathrm{n}} \leq \mathrm{t} \leq \mathrm{t}_{\mathrm{n}+1} \\ \mathrm{w}_{\mathrm{n}+1}(\mathrm{t})=\mathrm{y}_{\mathrm{n}}\end{array}\right.$

Where, $x(s)= \begin{cases}\phi(s)=e^{\text {as }} \sin \left(\frac{\pi}{2} s\right) & \text { for } s \leq 0 \\ \eta(s) & \text { for } 0 \leq s \leq t_{n}\end{cases}$

We know for ordinary differential equation,

$$
\begin{aligned}
& \mathrm{y}^{\prime}(\mathrm{t})=\mathrm{f}(\mathrm{t}, \mathrm{y}(\mathrm{t})) \\
& \mathrm{y}\left(\mathrm{t}_{0}\right)=\mathrm{y}_{0}
\end{aligned}
$$

The implicit mid point method is given by

$\mathrm{y}_{\mathrm{n}+1}=\mathrm{y}_{\mathrm{n}}+\operatorname{hf}\left(\mathrm{t}_{\mathrm{n}}+\frac{\mathrm{h}}{2}, \frac{1}{2}\left(\mathrm{y}_{\mathrm{n}}+\mathrm{y}_{\mathrm{n}+1}\right)\right)$

Which computes an approximation to a definite integral.

We can integrate DDE in eq.(3), using Gaussian collocation method and the method is known as midpoint rule.

For Gaussian point $v=1$, the general eq.(2) takes the form, 
$\mathrm{y}_{\mathrm{n}+1}=\mathrm{y}_{\mathrm{n}}+\mathrm{hf}\left(\mathrm{t}_{\mathrm{n}}+\frac{\mathrm{h}}{2}, \frac{\mathrm{y}_{\mathrm{n}}+\mathrm{y}_{\mathrm{n}+1}}{2}, \mathrm{x}\left(\mathrm{t}_{\mathrm{n}}+\frac{\mathrm{h}}{2}-1\right)\right)$

$\Rightarrow \mathrm{y}_{\mathrm{n}+1}=\mathrm{y}_{\mathrm{n}}+\mathrm{hf}\left(\mathrm{t}, \mathrm{w}_{\mathrm{n}+1}(\mathrm{t}), \mathrm{x}(\mathrm{t}-1)\right)$

Where $\mathrm{t}_{\mathrm{n}}+\frac{\mathrm{h}}{2}=\mathrm{t}, \frac{1}{2}\left(\mathrm{y}_{\mathrm{n}}+\mathrm{y}_{\mathrm{n}+1}\right)=\mathrm{w}_{\mathrm{n}+1}(\mathrm{t})$

Using eq.(5) to eq.(4), with constant integration step size $\mathrm{h}=\frac{1}{\mathrm{~m}-\delta}, \mathrm{m} \geq 2$ is an integer, $0 \leq \delta \leq 1$ gives

$$
\begin{aligned}
& y_{n+1}=\left\{\begin{array}{l}
y_{n}+h\left(a\left(\frac{y_{n}+y_{n+1}}{2}\right)-\frac{\pi}{2} e^{a} \phi\left(t_{n}+\frac{h}{2}-1\right)\right), \quad t_{n}+\frac{h}{2}-1 \leq 0 \\
y_{n}+h\left(a\left(\frac{y_{n}+y_{n+1}}{2}\right)-\frac{\pi}{2} e^{a} \eta\left(t_{n}+\frac{h}{2}-1\right)\right), \quad t_{n}+\frac{h}{2}-1>0
\end{array}\right. \\
& \because \mathrm{x}(\mathrm{s})=\left\{\begin{array}{ll}
\phi(\mathrm{s}) & \text { for } \mathrm{s} \leq 0 \\
\eta(\mathrm{s}) & \text { for } 0 \leq \mathrm{s} \leq \mathrm{t}_{\mathrm{n}}
\end{array} \text { and } \mathrm{s}=\mathrm{t}-1, \mathrm{t}=\mathrm{t}_{\mathrm{n}}+\frac{\mathrm{h}}{2},\right.
\end{aligned}
$$

Where $\eta\left(t_{n}+\frac{h}{2}-1\right)$ is given by linear interpolation

i.e, $\eta\left(\mathrm{t}_{\mathrm{n}}+\frac{\mathrm{h}}{2}-1\right)=\eta\left(\mathrm{t}_{\mathrm{n}}+\frac{\mathrm{h}}{2}-(\mathrm{m}-\delta) \mathrm{h}\right), \mathrm{h}=\frac{1}{(\mathrm{~m}-\delta)}$

$$
= \begin{cases}\left(\frac{1}{2}-\delta\right) y_{n-m}+\left(\delta+\frac{1}{2}\right) y_{n-m+1}, & 0 \leq \delta \leq \frac{1}{2} \\ \left(\frac{3}{2}-\delta\right) y_{n-m+1}+\left(\delta-\frac{1}{2}\right) y_{n-m+2}, & \frac{1}{2}<\delta<1\end{cases}
$$

$\because 0<\delta<1$ and $\frac{1}{2}-\delta+\delta+\frac{1}{2}=1, \frac{3}{2}-\delta+\delta-\frac{1}{2}=1$

As $\mathrm{t}_{\mathrm{n}} \leq \mathrm{t} \leq \mathrm{t}_{\mathrm{n}+1}$ and step size is $\mathrm{h}$,

$$
\text { So } \mathrm{t}_{\mathrm{n}}=\mathrm{nh}
$$

Also, $\mathrm{t}_{\mathrm{n}}+\frac{\mathrm{h}}{2}-1 \leq 0$

$\Rightarrow \mathrm{nh}+\frac{\mathrm{h}}{2}-1 \leq 0$

$\Rightarrow \mathrm{n}\left(\frac{1}{\mathrm{~m}-\delta}\right)+\frac{1}{2(\mathrm{~m}-\delta)}-1 \leq 0$

$$
\left(\because \mathrm{h}=\frac{1}{\mathrm{~m}-\delta}, 0 \leq \delta \leq 1\right)
$$

$\Rightarrow \frac{2 \mathrm{n}+1-2(\mathrm{~m}-\delta)}{2(\mathrm{~m}-\delta)} \leq 0$

$\Rightarrow 2 \mathrm{n}+1-2 \mathrm{~m}+2 \delta \leq 0$

$\Rightarrow \mathrm{n} \leq \mathrm{m}-\delta-\frac{1}{2}$

Similarly, $\mathrm{t}_{\mathrm{n}}+\frac{\mathrm{h}}{2}-1>0$ 


$$
\Rightarrow \mathrm{n}>\mathrm{m}-\delta-\frac{1}{2} .
$$

Now,

$$
\begin{aligned}
y_{n+1}= \begin{cases}y_{n}+h\left(a\left(\frac{y_{n}+y_{n+1}}{2}\right)-e^{a} \frac{\pi}{2} \phi\left(t_{n}+\frac{h}{2}-1\right)\right), & t_{n}+\frac{h}{2}-1 \leq 0 \\
y_{n}+h\left(a\left(\frac{y_{n}+y_{n+1}}{2}\right)-e^{a} \frac{\pi}{2} \eta\left(t_{n}+\frac{h}{2}-1\right)\right), & t_{n}+\frac{h}{2}-1>0\end{cases} \\
= \begin{cases}y_{n}+\frac{h}{2} a y_{n}+\frac{h}{2} a y_{n+1}-h e^{a} \frac{\pi}{2} \phi\left(t_{n}+\frac{h}{2}-1\right), & t_{n}+\frac{h}{2}-1 \leq 0 \\
y_{n}+\frac{h}{2} a_{n}+\frac{h}{2} a y_{n+1}-h e^{a} \frac{\pi}{2} \eta\left(t_{n}+\frac{h}{2}-1\right), & t_{n}+\frac{h}{2}-1>0\end{cases}
\end{aligned}
$$

For $\quad t_{n}+\frac{h}{2}-1 \leq 0$

$$
\begin{aligned}
& y_{n+1}=\left(1+\frac{h}{2} a\right) y_{n}+\frac{h}{2} a y_{n+1}-h e^{a} \frac{\pi}{2} e^{a\left(t_{n}+\frac{h}{2}-1\right)} \sin \left(\frac{\pi}{2}\left(t_{n}+\frac{h}{2}-1\right)\right) \\
& \Rightarrow y_{n+1}-\frac{h}{2} a y_{n+1}=\left(1+\frac{h}{2} a\right) y_{n}-\frac{\pi}{2} h e^{a\left(t_{n}+\frac{h}{2}\right)} \sin \left(\frac{\pi}{2}\left(t_{n}+\frac{h}{2}-1\right)\right) \\
& \Rightarrow\left(1-\frac{1}{2} h a\right) y_{n+1}=\left(1+\frac{1}{2} h a\right) y_{n}-\frac{\pi}{2} h e^{a\left(t_{n}+\frac{h}{2}\right)} \sin \left(\frac{\pi}{2}\left(t_{n}+\frac{h}{2}-1\right)\right) \\
& \Rightarrow y_{n+1}=\frac{\left(1+\frac{1}{2} h a\right) y_{n}-\frac{\pi}{2} h e^{a\left(t_{n}+\frac{h}{2}\right)} \sin \left(\frac{\pi}{2}\left(t_{n}+\frac{h}{2}-1\right)\right)}{1-\frac{1}{2} h a} \\
& \Rightarrow y_{n+1}=\frac{\left(1+\frac{1}{2} h a\right) y_{n}-\frac{\pi}{2} h e^{a\left(n h+\frac{h}{2}\right)} \sin \left(\frac{\pi}{2}\left(n h+\frac{h}{2}-1\right)\right)}{1-\frac{1}{2} h a} \\
& \Rightarrow y_{n+1}=\frac{\left(1+\frac{1}{2} h a\right) y_{n}-\frac{\pi}{2} h e^{a\left(n+\frac{1}{2}\right) h} \sin \left(\frac{\pi}{2}\left(\left(n+\frac{1}{2}\right) h-1\right)\right)}{1-\frac{1}{2} h a}
\end{aligned}
$$

For $t_{n}+\frac{h}{2}-1>0$

$$
\mathrm{y}_{\mathrm{n}+1}=\mathrm{y}_{\mathrm{n}}+\frac{\mathrm{h}}{2} \mathrm{ay}_{\mathrm{n}}+\frac{\mathrm{h}}{2} \mathrm{ay}_{\mathrm{n}+1}-\mathrm{he}^{\mathrm{a}} \eta\left(\mathrm{t}_{\mathrm{n}}+\frac{\mathrm{h}}{2}-1\right)
$$




$$
\begin{aligned}
& \Rightarrow \mathrm{y}_{\mathrm{n}+1}-\frac{1}{2} \text { hay }_{\mathrm{n}+1}= \begin{cases}\left(1+\frac{1}{2} \mathrm{ha}\right) \mathrm{y}_{\mathrm{n}}-\mathrm{he}^{\mathrm{a}} \frac{\pi}{2}\left[\left(\frac{1}{2}-\delta\right) \mathrm{y}_{\mathrm{n}-\mathrm{m}}+\left(\delta+\frac{1}{2}\right) \mathrm{y}_{\mathrm{n}-\mathrm{m}+1}\right], & 0 \leq \delta \leq \frac{1}{2} \\
\left(1+\frac{1}{2} \mathrm{ha}\right) \mathrm{y}_{\mathrm{n}}-\mathrm{he}^{\mathrm{a}} \frac{\pi}{2}\left[\left(\frac{3}{2}-\delta\right) \mathrm{y}_{\mathrm{n}-\mathrm{m}+1}+\left(\delta-\frac{1}{2}\right) \mathrm{y}_{\mathrm{n}-\mathrm{m}+2}\right], & \frac{1}{2}<\delta<1\end{cases} \\
& \Rightarrow\left(1-\frac{1}{2} \text { ha }\right) \mathrm{y}_{\mathrm{n}+1}= \begin{cases}\left(1+\frac{1}{2} \mathrm{ha}\right) \mathrm{y}_{\mathrm{n}}-\mathrm{he}^{\mathrm{a}} \frac{\pi}{2}\left[\left(\frac{1}{2}-\delta\right) \mathrm{y}_{\mathrm{n}-\mathrm{m}}+\left(\delta+\frac{1}{2}\right) \mathrm{y}_{\mathrm{n}-\mathrm{m}+1}\right], & 0 \leq \delta \leq \frac{1}{2} \\
\left(1+\frac{1}{2} \mathrm{ha}\right) \mathrm{y}_{\mathrm{n}}-\mathrm{he}^{\mathrm{a}} \frac{\pi}{2}\left[\left(\frac{3}{2}-\delta\right) \mathrm{y}_{\mathrm{n}-\mathrm{m}+1}+\left(\delta-\frac{1}{2}\right) \mathrm{y}_{\mathrm{n}-\mathrm{m}+2}\right], & \frac{1}{2}<\delta<1\end{cases} \\
& \Rightarrow \mathrm{y}_{\mathrm{n}+1}= \begin{cases}\frac{\left(1+\frac{1}{2} h \mathrm{ha}\right) \mathrm{y}_{\mathrm{n}}-\mathrm{he}^{\mathrm{a}} \frac{\pi}{2}\left[\left(\frac{1}{2}-\delta\right) \mathrm{y}_{\mathrm{n}-\mathrm{m}}+\left(\delta+\frac{1}{2}\right) \mathrm{y}_{\mathrm{n}-\mathrm{m}+1}\right]}{1-\frac{1}{2} \mathrm{ha}}, & 0 \leq \delta \leq \frac{1}{2} \\
\frac{\left(1+\frac{1}{2} h a\right) \mathrm{y}_{\mathrm{n}}-\mathrm{he}^{\mathrm{a}} \frac{\pi}{2}\left[\left(\frac{3}{2}-\delta\right) \mathrm{y}_{\mathrm{n}-\mathrm{m}+1}+\left(\delta-\frac{1}{2}\right) \mathrm{y}_{\mathrm{n}-\mathrm{m}+2}\right]}{1-\frac{1}{2} \mathrm{ha}}, & \frac{1}{2}<\delta<1\end{cases}
\end{aligned}
$$

Summing up the midpoint rule for equation (3), we get

$$
y_{n+1}=\frac{\left(1+\frac{1}{2} h a\right) y_{n}-\frac{\pi}{2} h e^{a\left(n+\frac{1}{2}\right) h} \sin \left(\frac{\pi}{2}\left(\left(n+\frac{1}{2}\right) h-1\right)\right)}{1-\frac{1}{2} h a}
$$

for $\mathrm{n} \leq \mathrm{m}-\delta-\frac{1}{2}$ and

$$
\mathrm{y}_{\mathrm{n}+1}= \begin{cases}\frac{\left(1+\frac{1}{2} h a\right) \mathrm{y}_{\mathrm{n}}-\frac{\pi}{2} \mathrm{e}^{\mathrm{a}} \mathrm{h}\left[\left(\frac{1}{2}-\delta\right) \mathrm{y}_{\mathrm{n}-\mathrm{m}}+\left(\delta+\frac{1}{2}\right) \mathrm{y}_{\mathrm{n}-\mathrm{m}+1}\right]}{1-\frac{1}{2} \mathrm{ha}}, & 0 \leq \delta \leq 1 \\ \frac{\left(1+\frac{1}{2} \mathrm{ha}\right) \mathrm{y}_{\mathrm{n}}-\frac{\pi}{2} \mathrm{e}^{\mathrm{a}} \mathrm{h}\left[\left(\frac{3}{2}-\delta\right) \mathrm{y}_{\mathrm{n}-\mathrm{m}+1}+\left(\delta-\frac{1}{2}\right) \mathrm{y}_{\mathrm{n}-\mathrm{m}+2}\right]}{1-\frac{1}{2} \mathrm{ha}}, & \frac{1}{2}<\delta<1\end{cases}
$$

for $\mathrm{n}>\mathrm{m}-\delta-\frac{1}{2}$

for $\mathrm{v}>1$ the method becomes more complicated whose solutions involve in linear system in R. More generally we shall see that for arbitrary step sizes in DDE method based on v-stage Gaussian collocation exhibit nodal and uniform accuracy orders equals to uniform order $v+1$ of the continuous ODE method.

The nodal accuracy of order $\mathrm{p}$ the resulting DDE Method considered above for its solution in the interval and this solution is of class $\mathrm{C}^{\infty}$. 
Hence no discontinuities are present which can spoiled accuracy order of the method.

\section{Conclusion:-}

In most applications of delay differential equation in the sciences, the need of including time delays is often due to the presence of process of times or the existence of stage structure.

1. In engineering applications, such time delays are often modeled via high dimensional compartment models.

2. In life-science applications, compartmental models can present the additional challenges of estimating some of the involved parameter values. In such cases, low-dimensional delay differential models with fewer parameters can be sensible alternatives.

Numerically solving most Delay Differential Equations (DDEs) or systems is almost as simple as solving Ordinary Differential Equations (ODEs).

In this paper we have given comparative study of DDEs and ODEs. In some cases, DDEs are equivalent to a system of ODEs, discussed in some examples. Further the characteristic equation associated with linear DDEs with discrete delays have been discussed. Lastly we have discussed the order failure in the comparison of DDEs versus ODEs.

\section{References:-}

1. Bellen Alfredo, Zennaro Marino(2003), Numerical methods for Delay Differential Equations, Oxford Science publications.

2. Belleman, Richard; Cooke, Kenneth L.(1963). Differential-difference equations. New York-London : Academic Press.

3. Briat, Corentin(2015), Linear Parameter-Varying and Time -Delay systems. Analysis, observation, filtering control. Springer Verlag Heidelberg. ISBN 978-662-44049-0.

4. Driver, Rodney D.(1977). Ordinary and Delay Differential Equations, New York : Springer verlag. ISBN 0387-90231-7.

5. Kuang, Y. 1993. Delay Differential Equations with applications in population dynamics, Boston : Academic Press.

6. Smith, H.L.2011. An introduction to delay differential equations with applications to the life sciences. Texts in applied mathematics, New York : Springer. 